\title{
Real-time Workshop Information Acquisition and Monitoring System Based on Three-Layer Network
}

\author{
Junhong Wang \\ Department of Electronic Engineering .North China \\ Institute of Aerospace Engineering \\ NCIAE \\ Langfang, China \\ e-mail: wang20050816@163.com
}

\section{Zongrui Li}

Department of Electronic Engineering .North China Institute of Aerospace Engineering

NCIAE

Langfang, China

e-mail: lizongrui@163.com

\author{
Donghui Sun \\ Department of Electronic Engineering .North China \\ Institute of Aerospace Engineering \\ NCIAE \\ Langfang, China \\ e-mail: lfsdh2000@126.com \\ Zhenzhong Xu \\ Department of Electronic Engineering .North China \\ Institute of Aerospace Engineering \\ NCIAE \\ Langfang, China \\ e-mail: xzz50@163.com
}

\begin{abstract}
The paper discussed the realization of workshop information acquisition and monitoring system which had three-layer network construction. According to analyzing the production information of the real-time monitoring workshop, raised the management network based on Ethernet which could manage order and tasks, the transmission network of data transmission, and the device network which was responsible for collecting real-time data. This system defined the management layer network communication protocol. The transmission layer and the device network use the standard protocol. The results indicate that the system realizes real-time data acquisition and workshop production of dynamic monitoring, ensures the efficient, safe, and orderly production.
\end{abstract}

Keywords-data acquisition; network; monitoring; protocol

\section{INTRODUCTION}

Production process in manufacturing enterprise workshop involves more and more complex equipment and process factors, in which situations and status information is complicated and changeable. This is due to the lack of information monitoring method in the traditional production workshop, so that the production system will appear in a variety of problems: such as lack of quantitative relationship between with workstations load, which won't lead balance production with the changed production target and conditions; such as without production information monitoring, fault event can't be processed, production has lack of guidance, workstation connected poor will lead to production blocking, the lack of material events will have occurred; At the same time, the main data based on manual reporting, whose time interval is large, easily influenced by artificial factors, can not guarantee the accuracy and real-time.

To realize the automation of manufacturing processes, in addition to system control and management technology etc, information acquisition process is very important. Only by adopting information acquisition technology, it can acquire state information of the manufacturing process fully, real-time, accurately. And further more, on this basis it can not only realize the effective control and management of the manufacturing process, but also improve the flexibility, robustness and fault handling capacity of the whole system. In the design the sensor technology, fieldbus technology and Ethernet technology is combined organically. Through the sensor it can collect workshop data information, by Fieldbus application this information is transmitted to the host computer of the central node. By monitoring software running in the computer, real time motion of the whole workshop assembly line is dynamic monitoring.

\section{The System Design PRINCIPLE}

It is the core task of workshop control system that production management and dispatching departments realize the dynamic scheduling. The traditional scheduling methods consider mostly some dynamic factors as a static in the running process of the system, so the practicability is very poor. With engineering research of manufacturing systems made in-depth, state feedback character become one of the characteristics of manufacturing system. During the manufacturing system operation, the output state such as product quality information and manufacturing resources utilization, constantly are given feedback to each link of the manufacturing process. Therefore, the manufacturing system achieves continuous adjustment, improvement and optimization. In the current shop floor control system research, the feedback information has become one of the essential parts, whose main function is to monitor running state and analyze data workshop for production management department.

Production data information is as follow: equipment working state, material flow state, quality inspection information etc. The data acquisition system not only collects production information, but also can issue production planning from the management layer to 
the bottom layer. It is mainly reflected in the following aspects:

- It is real-time data acquisition, including procedure schedule, material status, quality inspection information, tools and equipment state information etc. In the collection status, the related information feed back the production conditions in time, which provides timely and reliable data support for management decision.

- The production plans issued is achieved, which consist of the station information, process route, parts list, schedule, production command information etc. These make sure that the workers can view the information timely.

- This system can process all kinds of alarm information.

- Data transmit fast, accurately, and remote communication requirements can be realized.

To collect workshop information real-time, accurately, and high speed, intelligent sensors are essential. Application of intelligent sensor can realize real-time information collection. The information has the workshop equipment working state, production status, operation of equipment, personnel position operation content, production quantity, quantity of waste products, raw materials and so on.

By using fieldbus technology, all field sensors or actuator and other equipment connect to the controller, which formed a digital communication network at the workshop level. When performing data acquisition, intelligent sensor with standard fieldbus interface, will convert the detected signal to digital signal processed. Digital signal will replace the original standard analog signal. Information communication and transmission through the fieldbus and computer is possible. When implementing control, the host computer will send command information through the fieldbus to intelligent implementation part with standard fieldbus interface.

The digital and intelligent instrument network system based on fieldbus technology is the intelligent monitoring system, which is composed of various digital intelligent sensors, intelligent actuator and a level three network system. This complete digital intelligent network system can be connected with the management application terminal via Ethernet, achieve the management instruction issued and field data upload.

\section{The Hardware Design Of System Structure}

The data acquisition and monitoring system is composed of three parts: data acquisition layer, data transmission layer, data management layer. Wherein the data acquisition layer will achieve the real-time acquisition of various intelligent sensor for field data; the data transmission layer will accomplish real-time transmission of data by using fieldbus technology; the data management layer will process the real-time data and make decisions, issue all kinds of control instructions. The hardware structure of the system is as shown in Figure 1.

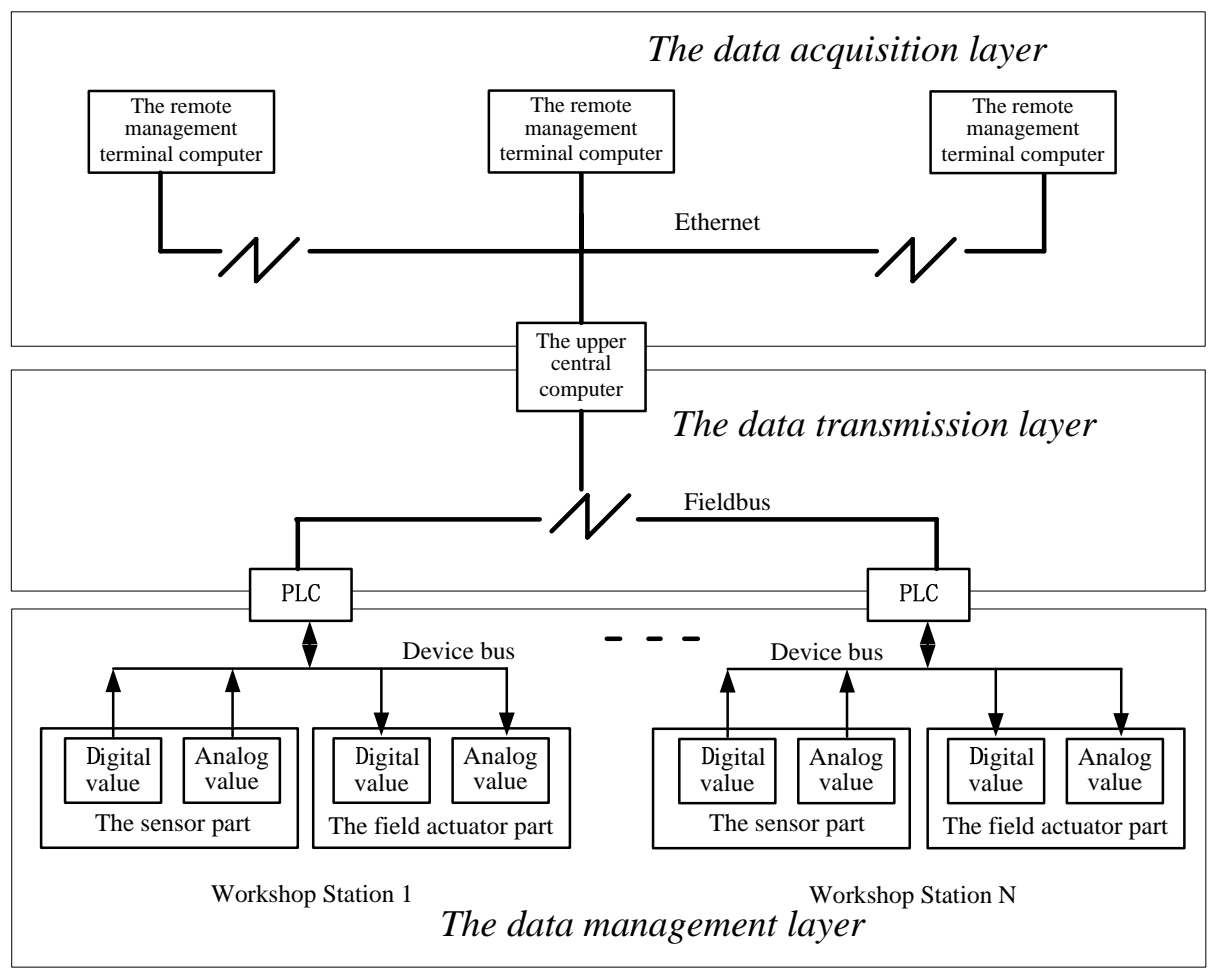

Figure 1. The hardware structure of the system. 
The system consists of three-level network, which is based on intelligent sensor technology, fieldbus technology and Ethernet Technology. All kinds of scene information acquisition sensors are connected together with the management terminal effectively, so that it can realize the real-time field information sharing and management instruction issued.

By the fieldbus technology, a high reliability data communication circuit is created in the control field, so as to realize the data communication between one intelligent sensor and the other intelligent sensor, between the intelligent sensor and main control machine. The main control computer regarded as the core is a IPC(Industrial Personal Computer), which monitor the actual network. Fieldbus as a link, the main control system can change the intelligent sensor single dispersed into network nodes, and gather preprocess data from the bottom intelligent sensor to the host through the fieldbus. Thus every intelligent sensor is connected to exchange information each other, the network control system is becoming which can complete control tasks together. It improves the accuracy, timely, rapidity of the entire network system.

\section{A. The data acquisition layer}

The data collection layer includes all kinds of intelligent sensor, standard bus and PLC. As the core of data acquisition, sensor is the basic unit of sensor networks, which carry on the acquisition and processing of various types of data. Standard sensor bus belongs to the field device bus, which can realize data transmission and instruction between sensors and a programmable controller. As the core of the whole system, programmable controller can collect all kinds of sensor information collected through the fieldbus, in which data transmission with the center computer is realized by fieldbus. It realizes multi node, real time, high speed data transmission.

\section{B. The data transmission layer}

The data transmission layer mainly comprises PLC, fieldbus and a host computer. This layer can exchange information between data information collected and host computer, ensure reliable and real-time transmission.

The fieldbus has the characteristics of open, digital, bidirectional, multi node, using serial communication mode. Due to the digital signal instead of analog signal, it has become a reality to improve system reliability, accuracy and anti-jamming ability, and to extend the transmission distance information. Each node of the fieldbus network has self diagnosis, self maintenance and control functions, following the unified standards and specifications, and having the ability of bidirectional communication. By fieldbus, without going to the equipment spot, maintenance personnel can inquire equipment status query on the operation, and control software download, setting, running condition adjustment and maintenance work.

\section{The data management layer}

The data management layer mainly includes the upper center computer, Ethernet, remote management terminal computer. This layer is responsible for information interaction between the remote management terminal computer and the computer of administration center. It is in charge of the production data transmission from the center computer to the remote management terminal. Through this layer, the center computer receives and processes the feedback data, and receives the instruction from remote management computer instructions.

Through the design of hardware structure, the system can realize the direct interaction between field data and information system, ensure the production tasks and feedback information timely delivery, timely find fault and alarm, and thus maximize production efficiency, safety requirements.

The programmable controller adopts S7-300 of the siemens company. The fieldbus is based on PROFIBUS bus. The host computer is an IPC, in which using the twisted pair Ethernet access. The remote terminal computer can use computer or PC terminal.

\section{THE SOFTWARE DESIGN OF THE SYSTEM}

\section{A. The function of the system}

The workshop production information acquisition and monitoring system mainly realize the following functions:

- The production of information analysis and coding, including the artifacts monitoring (current position and assembly state etc), the posts condition monitoring (the running state of the equipment and emergency requests etc), production process monitoring (production logistics state, each production unit to complete parts of the variety and quantity etc), fault monitoring.

- Information acquisition based on fieldbus.

- Using of high-level language programming to realize supervisory control and data acquisition.

B. The software structure of the system

The software structure consists of three parts:

- The data acquisition layer, in which the programmable controller collects the field data by bus. The controller uses programmable language, and the main flow chart is shown in figure 2.

- The data transmission layer, in which mainly data exchange between the programmable controller and the host computer. The programmable controller uses the programming language, and the central computer uses $\mathrm{C}++$ advanced language.

- The data management layer, in which mainly through the Ethernet, data is exchanged between the upper center computer and remote management computer terminal by using the advanced $\mathrm{C}++$ language. In this part of the software, data communication protocol is customized, in order to achieve the distinction between data and instructions. Thus data interaction is more timely and reliable. The data communication protocol command word is shown in Figure 3, and the data word is shown in figure 4.

Among them: "AA55" "55AA" is the initial word. ID is the terminal computer node number of the 8 correspondences, which is unique for the corresponding. "Command" is a command code of transmission between the management computer terminal and host computer. "The data length" is the subsequent data words in length. "Data" is the management of the computer terminal and the host computer's data center. 


\section{CONCLUSION}

The system combines the current overall situation of manufacturing workshop, analyzes the requirement of data acquisition system, put forward the overall design, adopts the three layer network, constructs the data acquisition system of manufacturing workshop. In the process of system development, the method of combining software and hardware is used. Considering practical and low cost considerations, the system applies programmable controller as the core collected in the field, therefore not only can achieve the required function, but also reduce the cost of data acquisition device. By applying supervisory control and data acquisition system, all kinds of information of workshop production line can be quickly and accurately transmitted to the monitoring and management system, so that the system can make timely reaction. The system is running stably, which has improved the production efficiency and achieved good results.

\section{ACKNOWLEDGMENT}

The study is based on the project of education department, whose number is z2013145. This work is financially supported by North China Institute of Aerospace Engineering, based on the program of key discipline "Detection Technology and Automatic equipment" and the emphasis development disciplines "Signal and information processing" of Hebei Province.

\section{REFERENCES}

[1] Fu Pinxin,Wang Meilin,Dai Qingyun,Zhong Runyang, "Research and application of workshop real-time information network of three-layer architecture,” Modern Manufacturing Engineering[J], 2012, No.04, pp.21-25.

[2] Yao Bin,"Research on data acquisition and monitoring system of CSS three layer architecture based on Ethernet Technology,” China New Technologies and Products[J],2009, No.05, pp.13.

[3] Fu Pinxin,Hu Guojin,Dai Qingyun,Wang Meilin,ZhongRunyang, "Research and Application of Workshop Real-Time Information Network of Four-Layer Architecture,” Measurement \& Control Technology[J], 2012, No.12, pp.82-85.

[4] Wang Wenlei, Xu Tingrong, "The Studying And Realizing Of Communication Technology Between PLC And PC," Micro computer information[J], vol 22.5-1, 2006, pp.43-46.

[5] Li Kaiyuan, “Application of PROFIBUS-DP Field Bus Technology to Steel Pipe Transmission System,”STEEL PIPE[J], Jun, 2013, Vol 42, No.3, pp.67-70.

[6] Hu Huiyu, "Based on the industrial ethernet to realize the remote monitoring between the upper computer and PLC,'Journal of Changchun Institute of Technology[J], 2011, Vol.12, No.2, pp.113116.

[7] LI Zhi,WANG Hui-fen,LIU Ting-ting,FEI Yi-zheng, "Design of Workshop Real-Time Monitoring System for Manufacturing Process,” Machinery Design \& Manufacture[J], 2013, No.03, pp.256-259.

[8] Xue Yuan, Liu Weidong "Design and Realization of Multi-channel Real-time Data Acquisition and Monitoring System,” Computer Measurement \& Control[J], 2011, No.04, pp.863-865.

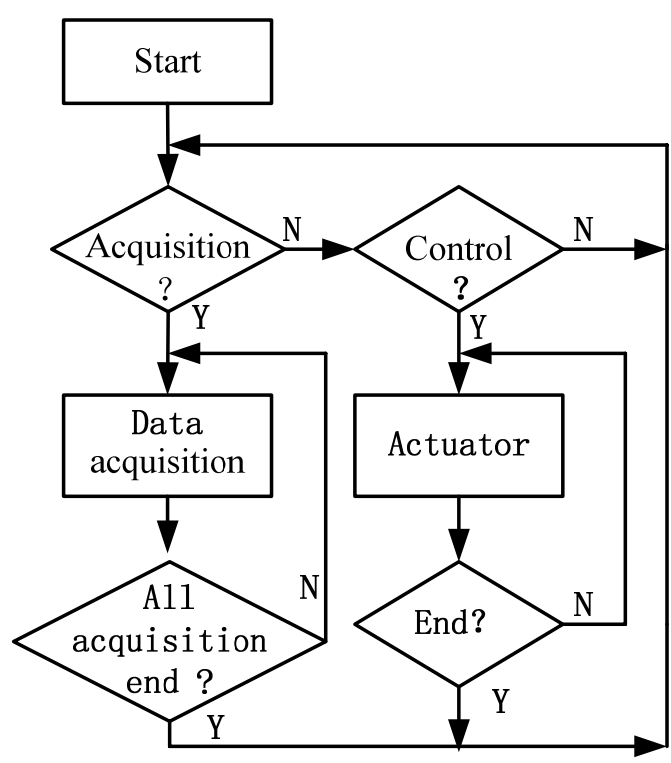

Figure2. The software flow chart of data acquisition part

\section{\begin{tabular}{l|l|l} 
AA55 & ID(8bits) & Command \\
\hline
\end{tabular}}

Figure3. The command word structure of data communication

55AA $\quad$ ID(8bits) $\quad$ Data length $\quad$ Data

Figure4. The data word structure of data communication 